\title{
ESTUDIO DE CUMPLIMIENTO DEL TRATAMIENTO ANTIDEPRESIVO
}

\section{A STUDY OF COMPLIANCE IN THE ANTIDEPRESSANT TREATMENT}

\author{
H. VARGAS-MURGA', J. VEGA-DIENSTMAIER ${ }^{2}$, J. CARPIO-TAPIA \\ W. LEYVA', C. MENDOZA', M. LUQUE' y G. MALÁSQUEZ.
}

\begin{abstract}
RESUMEN
Objetivo. Estudiar las variables asociadas al cumplimiento del tratamiento antidepresivo en un sector de la población peruana y comparar lo hallado con lo reportado en la literatura mundial.

Método. Se incluyeron 133 pacientes de consulta externa, vistos entre marzo y junio de 1999, correspondientes a los siguientes establecimientos de salud: Instituto Nacional de Salud Mental "Honorio Delgado-Hideyo Noguchi", Hospital Hipólito Unanue, Hospital María Auxiliadora y Clínica Ricardo Palma.

Resultados. Las variables que se asociaron significativamente con cumplimiento del tratamiento fueron: psicoterapia complementaria $(p<0.001)$, número de consultas $(p<0.001)$, explicación clara por parte del médico de cómo debía tomar la medicación ( $p<0.001)$, creencia de que el medicamento ayuda o es beneficioso ( $p<0.001)$, respuesta al tratamiento $(p=0.002)$, considerar que la medicación es necesaria para su tratamiento $(p=0.003)$, buen trato por parte del médico $(p=0.007)$, confianza en el médico tratante $(p=0.01)$ cefalea $(p=0.026)$ e insomnio $(p=0.041)$; por otro lado, los factores que se asociaron signi-ficativamente al incum-plimiento fueron: problemas para cubrir el costo de la medicación ( $p<0.001)$, temor a hacerse dependiente de la medicación $(p=0.003)$, considerar que es suficiente tomar la medicación sólo hasta sentirse bien ( $p=0.044)$ y dolor abdominal $(p=0.03)$. Los pacientes incumplidores consideraron que el costo de la medicación era la principal causa por la que habían dejado el tratamiento.
\end{abstract}

Conclusiones. Las variables asociadas al cumplimiento del tratamiento son similares a las encontradas en otros estudios, destacando las relacionadas con la información que el paciente recibe, la relación médico-paciente y el costo del fármaco.

Palabras-clave: Depresión. Tratamiento antidepresivo.

\footnotetext{
1. Médico Psiquiatra - Psiquiatra de Niños y Adolescentes, IESM "HD-HN", UPCH

2. Médico Psiquiatra. UPCH

3. Médico Psiquiatra, HHU, UPCH.

4. Médico Psiquiatra. UNMSM.

5. Médico Psiquiatra. UPCH.

6. Médico Cirujano. UPCH:
} 


\begin{abstract}
Objective. To study the variables asociated with compliance to the antidepressant treatment in a sector of the Peruavian population and to compare the results to the findings reported in the world literature.

Method. We included 133 out-patients attended between March and June of 1999, from the next health center: National Institute of Mental Health "Honorio Delgado-Hideyo Noguchi", Hipólito Unanue Hospital, María Auxiliadora Hospital and Ricardo Palma Clinic.

Results. The variables significantly associated with the treatment compliance were: complementary psychotherapy $(p<0.001)$, number of visits $(p<0.001)$, clear explanation from the physician about how to take medication $(p<0.001)$, to believe that medication is helpful $(p<0.001)$, response to treatment $(p=0.002)$, to consider that medication is necessary for treatment ( $p=0.003$ ), good personal treatment from the physician ( $p=0.007)$, to trust in physician $(p=0.01)$, headache $(p=0.026)$ and insomnia $(p=0.041)$; in addition, factores significantly associated with incompliance were: problems to cover the cost of the medication $(p<0.001)$, fear to become dependent to medication, ( $p=0.003)$, to consider that is sufficient to take medication only until to feel fine $(p=0.044)$ and abdominal pain $(p=0.03)$. The non-compliant patients consider that the cost of medications was the main cause for the abandonment of therapy.

Conclusions. The variables associated with compliance of treatment are similar to that founded in other studies, being remarkable that related with the information received by the patient, the patient-physician relationship and the cost of medication.
\end{abstract}

Key wORDS: Depression. Antidepressant treatment.

\section{INTRODUCCIÓN}

La depresión es una enfermedad médica bastante frecuente y de curso crónico y recurrente. ${ }^{1}$ El impacto social de la depresión incluye tanto la invalidez inducida como la carga impuesta a los familiares. Diversos factores se han asociado como predictores de recaída o recurrencia de la depresión, entre los más importantes se mencionan: gravedad de los síntomas, historia de episodios previos, presencia de enfermedades concomitantes, abuso y dependencia de sustancias y el incumplimiento del tratamiento psicofarmacológico antidepresivo. ${ }^{2} \mathrm{El}$ término cumplimiento o adherencia se refiere al grado en que el paciente toma su medicación en la forma en que fue prescrita. ${ }^{3} \mathrm{El}$ incumplimiento del tratamiento es un problema frecuente en la práctica médica cotidiana. Se estima que entre $20 \%$ y $80 \%$ de pacientes en general no cumplen con la terapia indicada. ${ }^{4} \mathrm{El}$ incumplimiento de la farmacoterapia antidepresiva es bastante frecuente. ${ }^{1}$ Lit et al. ${ }^{5}$ encontraron que el $28 \%$ de los pacientes abandonan su medicación durante el primer mes de tratamiento y el $44 \%$ durante el tercero. El incumplimiento es un fenómeno multifactorial que incluye factores psicosociales y relacionados a la medicación, entre otros. ${ }^{6,78}$

\section{FACTORES PSICOSOCIALES}

La educación del paciente sobre la naturaleza de su enfermedad, la importancia de tomar la medicación y los efectos adversos de 
los fármacos es importante para una buena adherencia al tratamiento. ${ }^{1,9,10,11,12}$ Una buena relación médico-paciente, con el aporte psicoterapéutico que esto significa o la asociación de una adecuada psicoterapia, influyen favorablemente en el cumplimiento de la medicación antidepresiva y en el pronóstico de la depresión. ${ }^{13,14,15,16,17,18} \mathrm{El}$ incumplimiento puede ser más frecuente en determinadas culturas debido a creencias erróneas sobre la enfermedad mental y la necesidad de usar medicación para ésta. ${ }^{19}$

\section{FACTORES RELACIONADOS CON LA MEDICACIÓN}

Los efectos adversos de los antidepresivos y los esquemas terapéuticos complejos son factores importantes que afectan la adherencia al tratamiento. De acuerdo con Hasan et al. ${ }^{20}$ el cumplimiento puede mejorar si se reduce la complejidad del esquema de tratamiento con una sola dosis por día, que podría ser administrada hacia la noche o al ir a dormir, lo cual disminuiría además ciertos efectos adversos. Según Cohen et al. ${ }^{21}$ la reducción en el número de fármacos administrados, así como la reducción en la frecuencia de administración de la droga, favorecen el cumplimiento. Uno de los factores que más inciden en la deficiente adherencia a los antidepresivos son los efectos adversos que provocan. Aproximadamente el $20 \%$ de pacientes con depresión rechazan los medicamentos y un $50 \%$ de los que toman los abandonan a causa de determinados efectos adversos o actitudes negativas con respecto al tratamiento. ${ }^{6,7,8,9,22}$ Diversos autores señalan que los efectos colaterales intolerables son una causa de abandono del tratamiento antidepresivo, siendo mayor el porcentaje con los antidepresivos heterocíclicos (tricíclicos y tetracíclicos) que con los ISRS y los nuevos antidepresivos. ${ }^{23,24,25,26,27,28}$ Los efectos colaterales que se relacionan más con incumplimiento del tratamiento son: sedación, sequedad de boca, aumento de peso y disfunciones sexuales. ${ }^{29,30,31,32,33,34,35,36,37,38}$

Otros factores como la comorbilidad asociada a la depresión (distimia, desorden de ansiedad y abuso de sustancias) se relacionan también con abandono del tratamiento..$^{39,40,41}$ Igualmente se señalan: la severidad de los síntomas depresivos, ${ }^{42}$ la falta de soporte familiar y laboral, ${ }^{43,44}$ el libre consentimiento de recibir un tratamiento antidepresivo ${ }^{45} \mathrm{y}$ temor a hacerse dependiente de la medicación. ${ }^{46}$

Sin embargo, hay otros factores adicionales que podrían estar relacionados al incumplimiento del tratamiento como: falta de conciencia de enfermedad, aumento o disminución rápida de la dosis, inicio del tratamiento en la fase terminal del episodio depresivo, la polifarmacia (interacciones medicamentosas e ingestión de varios fármacos al día) y el costo de la mediación, que se desprenden de la práctica médica cotidiana.

El objetivo del presente estudio fue determinar las variables asociadas al cumplimiento del tratamiento antidepresivo en un sector de la población peruana y comparar lo hallado con lo reportado en la literatura mundial.

\section{MÉTODO}

Se incluyeron 133 pacientes de consulta externa, vistos entre marzo y junio de 1999 , los que correspondieron a los siguientes establecimientos de salud: Instituto Nacional de Salud Mental "Honorio Delgado-Hideyo Noguchi” (INSM “HD-HN"), Hospital Hipólito Unanue (HHU), Hospital Auxiliar María Auxiliadora (HAMA) y Clínica Ricardo Palma (CRP). Los pacientes del INSM "HD-HN", HHU y HAMA fueron captados 
en una de sus citas de seguimiento. Los pacientes de la CRP fueron captados a través de un programa de despistaje gratuito de la depresión. Se les llamó por teléfono meses después de haber iniciado el tratamiento, para una cita gratuita de seguimiento.

Los criterios de inclusión fueron los siguientes: edad mayor de 18 años, cumplimiento de los criterios del DSM-IV para el diagnóstico de depresión y/o distimia, y estar recibiendo tratamiento farmacológico. Los criterios de exclusión fueron: tener depresión secundaria a enfermedad médica o abuso de sustancias, depresión post psicótica, depresión bipolar y trastorno de adaptación con ánimo deprimido; y asimismo, comorbilidad médica importante que tenga alguna relación con la presencia de síntomas depresivos.

Se utilizó un formato de evaluación de cumplimiento del tratamiento antidepresivo, elaborado por los autores del trabajo, con el cual el paciente fue entrevistado, por un médico diferente a su médico tratante. Se definió incumplimiento del tratamiento antidepresivo cuando el tiempo en que había dejado de tomar la medicación del paciente excedió 2 veces la vida media de la droga o cuando la cantidad de medicación no tomada por semana excedía el $30 \%$ del total. Esto último, tomando como base los criterios usados en otros estudios de cumplimiento. ${ }^{46,47}$

\section{RESULTADOS}

\section{Características sociodemográficas}

Se estudió un total de 133 pacientes, de los cuales $103(77.4 \%)$ eran mujeres y 30 (22.6\%), varones. La mayor parte de la población $(96.2 \%)$ era de raza mestiza, existiendo $1.5 \%$ de blancos y $2.3 \%$ de raza negra. En cuanto al estado civil, $36.8 \%$ eran solteros;
$47.4 \%$, casados o convivientes; $12.8 \%$, divorciados o separados; y $3.0 \%$, viudos. La religión de los pacientes eran mayormente católica $(90.2 \%) ; 3.8 \%$ eran evangélicos y $6.0 \%$ pertenecían a otras religiones. En relación a su situación laboral, el 49.6\% estaban desocupados; el $19.5 \%$ tenían trabajo eventual y el $30.8 \%$, trabajo estable. La mayoría vivían con familiares o conocidos (94.7\%); $3.8 \%$ estaban en una institución y $1.5 \%$ vivían solos. La mayoría de pacientes habían nacido en el departamento de Lima (69.17\%) y procedían con mayor frecuencia de los distritos de San Martín de Porras (9.0\%), San Juan de Lurigancho (7.5\%), La Molina $(6.0 \%)$ y Villa María del Triunfo (6.0\%). Según la institución de procedencia, el 52.6\% de pacientes fueron de la CRP; el 20.3\%, del HHU; el $17.3 \%$, del INSM "HD-HN"; y el $9.8 \%$, del HAMA. El ingreso familiar mensual fue el siguiente: menos de 300 soles, $3 \%$; entre 301 y 600 soles, $21.8 \%$; entre 601 y 900 soles, $20.3 \%$; entre 901 y 1500 soles, $27.1 \%$; y más de 1500 soles, $27.8 \%$. El gasto mensual en medicinas fue el siguiente: menos de 50 soles, 6.8\%; entre 51 y 100 soles, $16.5 \%$; entre 101 y 200 soles, $65.4 \%$; entre 201 y 400 soles, $10.5 \%$; y más de 400 soles, $0.8 \%$. Dentro de las variables numéricas que se muestran en la tabla 1 se encuentran algunas de tipo sociodemográfico (la edad y el grado de instrucción).

\section{Características clínicas}

La severidad del último episodio fue considerada como leve en el 3.8\%, moderada en el $69.9 \%$ y severa en el $26.3 \%$. Según el tipo de depresión: $33 \%$ correspondió a depresión mayor - episodio único; $39.1 \%$, a depresión mayor recurrente; $4.5 \%$, a depresión psicótica; $8.3 \%$, a distimia; y $14.3 \%$ a depresión doble. Respecto a los antecedentes personales de otras enfermedades: $19.5 \%$ tenía enfermedad orgánica concomitante; $6.0 \%$, 
TABLA 1

CARACTERÍSTICAS DE LA POBlaCión (VARIABles NUMÉRICAS)*

\begin{tabular}{|l|c|c|c|r|}
\hline Variables numéricas & Mínimo & Máximo & Promedio & \multicolumn{1}{|c|}{ DE $\dagger$} \\
\hline Edad (años) & 18 & 76 & 38.64 & 13.95 \\
Instrucción (años de estudio) & 1 & 20 & 12.33 & 3.91 \\
Edad de inicio (años) & 4 & 80 & 35.21 & 15.24 \\
Tiempo de enfermedad (meses) & 1 & 468 & 46.94 & 64.87 \\
Número de episodios previos & 0 & 16 & 1.46 & 2.62 \\
Consultas desde que inició el tratamiento & 1 & 30 & 4.57 & 3.77 \\
Tiempo del episodio actual (meses) & 0 & 96 & 11.58 & 16.29 \\
Duración de tratamiento (semanas) & 0 & 164 & 12.91 & 17.21 \\
\hline
\end{tabular}

* Total de pacientes estudiados $=133$.

$\dagger \mathrm{DE}=$ desviación estándar.

trastorno de pánico; $6.0 \%$ alcoholismo; $6.0 \%$, trastorno de personalidad y $4.5 \%$, fobia social; y en cuanto a los antecedentes familiares se encontró depresión en $33.1 \%$, alcoholismo en $24.1 \%$ y esquizofrenia en $11.3 \%$. El $22.6 \%$ de los pacientes recibió psicoterapia en el último episodio y $1.5 \%$, rehabilitación. El 36.8\% había tenido tratamiento farmacológico anteriormente. En la tabla 1 aparecen también algunas variables numéricas de relevancia clínica. La tabla 2 muestra la frecuencia de los antidepresivos que recibían los pacientes. Respecto a otros fármacos utilizados, éstos fueron: ansiolíticos

TABLA 2

PSICOFÁRMACOS USADOS

\begin{tabular}{|l|c|r|}
\hline Psicofármaco & Frecuencia & \multicolumn{1}{|c|}{$\%$} \\
\hline Amitriptilina & 16 & 12.0 \\
Clomipramina & 3 & 2.3 \\
Trimipramina & 1 & 0.8 \\
Maprotilina & 1 & 0.8 \\
Trazodona & 1 & 0.8 \\
Fluoxetina & 38 & 28.6 \\
Paroxetina & 15 & 11.3 \\
Sertralina & 18 & 13.5 \\
Fluvoxamina & 18 & 13.5 \\
Citalopram & 1 & 0.8 \\
Moclobemida & 2 & 1.5 \\
Mirtazapina & 5 & 3.8 \\
Combinado & 9 & 6.8 \\
Otro & 5 & 3.8 \\
\hline Total & 133 & 100.0 \\
\hline
\end{tabular}


(45.9\%), hipnóticos (3.1\%), antipsicóticos $(2.3 \%)$ y otros $(50.4 \%)$. Sobre la respuesta al tratamiento: en $5.3 \%$ fue nula; en $15.0 \%$ hubo una leve mejoría; en $27.8 \%$, una mejoría moderada; en $34.6 \%$, una gran mejoría; y en $17.3 \%$, una remisión completa.

\section{Cumplimiento de la medicación}

De los 133 pacientes, 66 eran incumplidores (52 con recaída [39.1\%] y $14 \sin$ recaída [10.5\%]) y 67 eran cumplidores (64 sin recaída [48.1\%] y 3 con recaída [2.3\%]). El $55.6 \%$ de los pacientes no olvidaban ninguna toma de la medicación; dentro de las tomas que omitían, la más frecuente era la del desayuno (27.1\%); siendo los olvidados del almuerzo y la cena mucho menos frecuentes ( $1.5 \%$ y $0.8 \%$, respectivamente).

En la tabla 3 se muestran las causas de incumplimiento según los pacientes. De acuerdo a esto, la mayoría atribuyó el abandono del tratamiento al costo de la medicación. Las tablas 4, 5 y 6 muestran las relaciones entre diversas variables y el cumplimiento. Tomando en cuenta estos resultados, las variables asociadas al cumplimiento de los antidepresivos son: haber recibido psicoterapia en el último episodio, mayor respuesta al tratamiento, mayor gasto en medicación y más consultas desde el inicio del tratamiento. La tabla 7 muestra la asociación entre las preguntas del cuestionario y el cumplimiento. En la tabla 8 aparece la relación entre los efectos adversos de la medicación y el cumplimiento.

\section{DISCUSIÓN}

\section{Características sociodemográficas}

Según los resultados que hemos obtenido, no se encontró asociación entre cumplimiento del tratamiento antidepresivo y alguna de estas variables (tabla 4,5 y 6).

\section{Características clínicas y tratamiento}

Dentro de las variables estudiadas, solamente se encontró asociación con: psicoterapia asociada en el último episodio, respuesta al tratamiento y número de consultas desde el inicio del tratamiento (tabla 4 y 6). La asociación de una adecuada psicoterapia influyendo favorablemente en el cumplimiento

TABLA 3

CAUSAS DE INCUMPLIMIENTO SEGÚN LOS PACIENTES

\begin{tabular}{|l|r|r|}
\hline Causas de incumplimiento de la medicación según paciente & Frecuencia & \% \\
\hline Costo & 46 & 69.7 \\
Creer que no lo necesita & 4 & 6.1 \\
Efecto adverso & 2 & 3.0 \\
Médico le indicó que la deje & 2 & 3.0 \\
No disponible en farmacia & 1 & 1.5 \\
Sentirse mejor & 7 & 10.6 \\
Temor a dependencia & 2 & 3.0 \\
otro & 2 & 3.0 \\
\hline Total & 66 & 100.0 \\
\hline
\end{tabular}


TABLA 4

ASOCIACIÓN ENTRE VARIABLES CATEGÓRICAS DICOTÓMICAS Y CUMPLIMIENTO

\begin{tabular}{|l|c|cc|c|c|}
\hline \multicolumn{1}{|c|}{ Variable } & $\begin{array}{c}\text { \% con la } \\
\text { variable } \\
\text { presente }\end{array}$ & $\begin{array}{c}\text { \% de cumplimiento } \\
\text { entre los que } \\
\text { tuvieron la variable }\end{array}$ & P* & \multicolumn{1}{c|}{ OR } \\
(IC-95\%)
\end{tabular}

* Prueba Chi-cuadrado, excepto el valor marcado con $\dagger$

$\dagger$ Prueba de Fisher de 2 colas.

TABLA 5

RELACIÓN ENTRE VARIABLES NUMÉRICAS CON DISTRIBUCIÓN NORMAL Y CUMPLIMIENTO

\begin{tabular}{|l|c|c|c|}
\hline \multirow{2}{*}{ Variable } & \multicolumn{2}{|c|}{ Promedio (DE)* } & Prueba t \\
\cline { 2 - 4 } & Cumplidores & Incumplidores & $\mathrm{p}$ \\
\hline Edad & $39.24(13.93)$ & $38.03(14.05)$ & 0.619 \\
Edad de inicio & $35.31(15.61)$ & $35.11(14.97)$ & 0.938 \\
Años de estudio & $12.07(4.40)$ & $12.59(12.59)$ & 0.448 \\
\hline
\end{tabular}

* $\mathrm{DE}=$ desviación estándar.

TABLA 6

RELACIÓN ENTRE VARIABLES ORDINALES Y NUMÉRICAS SIN DISTRIBUCIÓN NORMAL Y CUMPLIMIENTO

\begin{tabular}{|l|c|c|c}
\hline \multirow{2}{*}{ Variable } & \multicolumn{2}{|c|}{ Lugar medio } & Prueba de Mann-Whitney \\
\cline { 2 - 4 } & Cumplidores & Incumplidores & $\mathrm{P}$ \\
\hline Ingreso familiar & 70.76 & 63.18 & 0.242 \\
Tiempo de enfermedad & 65.34 & 68.68 & 0.617 \\
Tiempo del episodio actual & 66.73 & 67.27 & 0.935 \\
$\begin{array}{l}\text { Número de episodios previos } \\
\text { Severidad de los síntomas }\end{array}$ & 63.61 & 70.44 & 0.273 \\
del último episodio & 69.64 & 64.32 & 0.319 \\
$\begin{array}{l}\text { Número de veces al día que } \\
\text { tiene indicada la medicación }\end{array}$ & 65.63 & 68.39 & 0.521 \\
Respuesta al tratamiento & 76.81 & 57.04 & 0.002 \\
$\begin{array}{l}\text { Gasto al mes en medicinas } \\
\text { Consultas desde inicio de }\end{array}$ & 72.95 & 60.96 & 0.034 \\
tratamiento & 79.78 & 54.02 & $<0.001$ \\
Duración del tratamiento & 71.74 & 62.19 & 0.152 \\
\hline
\end{tabular}


TABLA 7

ASOCIACIÓN ENTRE LAS PREGUNTAS DEL CUESTIONARIO Y CUMPLIMIENTO

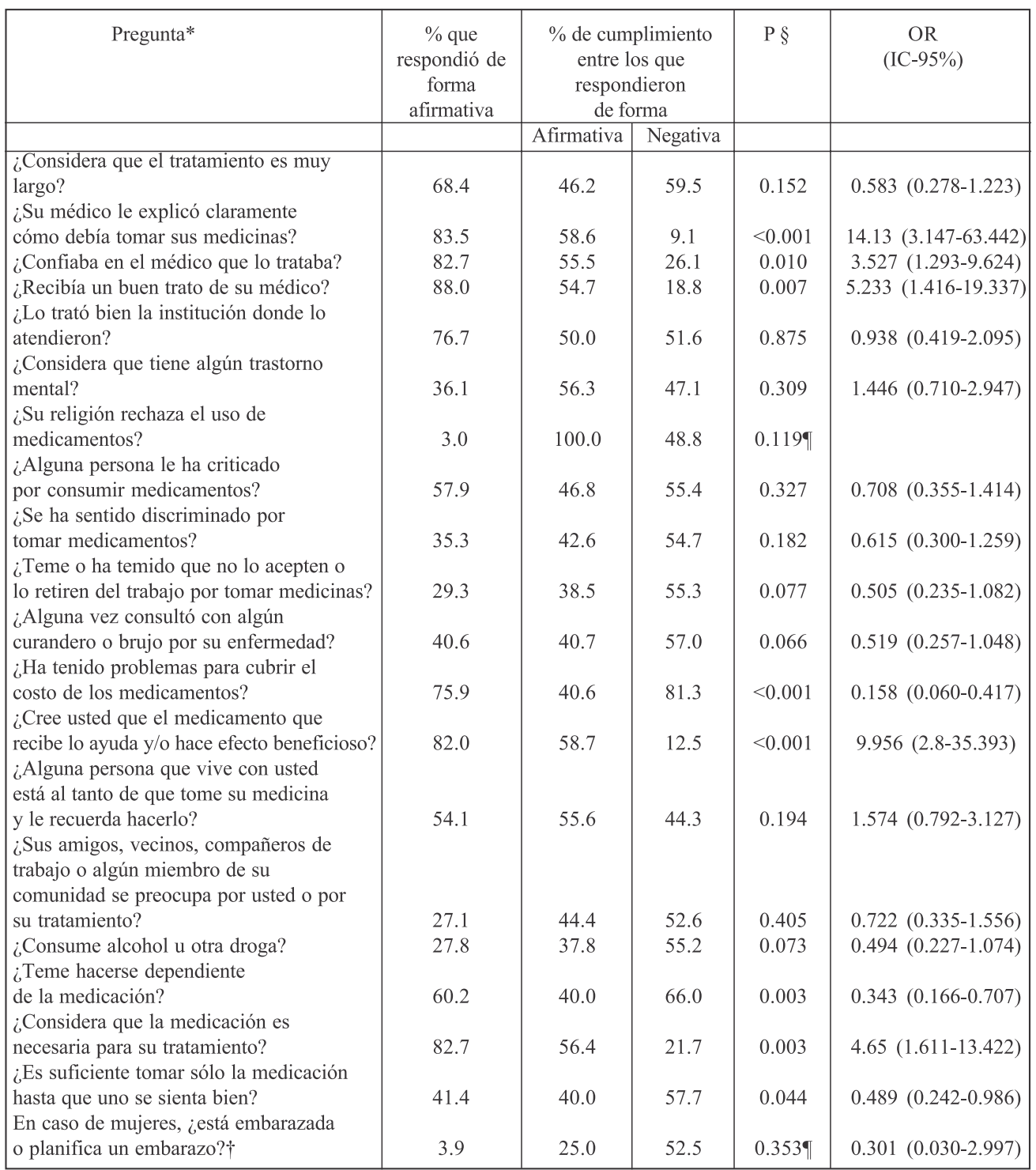

* Análisis realizado sobre el total de pacientes $(\mathrm{N}=133)$ a menos que se indique lo contrario con el símbolo $\dagger$.

† Análisis realizado con el total de mujeres $(\mathrm{N}=103)$.

$\S$ Chi cuadrado excepto los valores marcados con II.

II Prueba exacta de Fisher de 2 colas. 
TABLA 8

RELACIÓN ENTRE EFECTOS COLATERALES Y CUMPLIMIENTO DE LA MEDICACIÓN

\begin{tabular}{|c|c|c|c|c|c|}
\hline \multirow[t]{2}{*}{ Efectos Colaterales* } & \multirow[t]{2}{*}{$\begin{array}{l}\% \text { que } \\
\text { respondió de } \\
\text { forma } \\
\text { afirmativa } \\
\end{array}$} & \multicolumn{2}{|c|}{$\begin{array}{l}\% \text { de cumplimiento } \\
\text { entre los que } \\
\text { respondieron } \\
\text { de forma }\end{array}$} & \multirow[t]{2}{*}{$\mathrm{P} \S$} & \multirow[t]{2}{*}{$\begin{array}{c}\text { OR } \\
(\mathrm{IC}-95 \%)\end{array}$} \\
\hline & & Afirmativa & Negativa & & \\
\hline Secado de boca & 48.1 & 51.6 & 49.3 & 0.792 & $1.096(0.555-2.164)$ \\
\hline Visión borrosa & 24.8 & 63.6 & 46.0 & 0.079 & $2.054(0.913-4.623)$ \\
\hline Cefalea & 39.8 & 62.3 & 42.5 & 0.026 & $2.232(1.097-4.543)$ \\
\hline Alteración de la memoria & 26.3 & 60.0 & 46.9 & 0.185 & $1.696(0.774-3.714)$ \\
\hline Estreñimiento & 27.8 & 45.9 & 52.1 & 0.526 & $0.782(0.366-1.673)$ \\
\hline Retención urinaria & 10.5 & 64.3 & 48.7 & 0.271 & $1.893(0.599-5.984)$ \\
\hline Obstrucción intestinal & 0.8 & 0.0 & 50.8 & 0.4969 & - \\
\hline Aumento de peso & 42.1 & 57.1 & 45.5 & 0.183 & $1.6(0.799-3.202)$ \\
\hline Aumento de apetito & 40.6 & 55.6 & 46.8 & 0.323 & $1.419(0.708-2.844)$ \\
\hline Disminución de peso & 18.8 & 48.0 & 50.9 & 0.792 & $0.890(0.372-2.124)$ \\
\hline Disminución de apetito & 21.1 & 50.0 & 50.5 & 0.964 & $0.981(0.426-2.258)$ \\
\hline Somnolencia & 53.4 & 50.7 & 50.0 & 0.935 & $1.029(0.520-2.033)$ \\
\hline Insomnio & 29.3 & 64.1 & 44.7 & 0.041 & $2.211(1.023-4.776)$ \\
\hline Mareos & 32.3 & 51.2 & 50.0 & 0.900 & $1.048(0.506-2.167)$ \\
\hline Hipotensión (presión baja) & 12.8 & 47.1 & 50.9 & 0.770 & $0.859(0.310-2.380)$ \\
\hline $\begin{array}{l}\text { Sensación de que uno mismo o las } \\
\text { cosas dan vueltas (vértigo) }\end{array}$ & 14.3 & 47.4 & 50.9 & 0.777 & $0.869(0.329-2.298)$ \\
\hline Palpitaciones o taquicardia & 21.8 & 51.7 & 50.0 & 0.870 & $1.071(0.470-2.441)$ \\
\hline Ansiedad (sentirse "nervioso") & 36.1 & 43.8 & 54.1 & 0.251 & $0.659(0.323-1.344)$ \\
\hline Acatisia & 5.3 & 57.1 & 50.0 & 1.0009 & $1.333(0.287-6.202)$ \\
\hline Náuseas y/o vómitos & 33.1 & 45.5 & 52.8 & 0.425 & $0.745(0.361-1.537)$ \\
\hline Dolor abdominal & 12.0 & 25.0 & 53.8 & 0.030 & $0.286(0.087-0.938)$ \\
\hline Diarrea & 3.8 & 60.0 & 50.0 & 1.0009 & $1.50(0.242-9.280)$ \\
\hline Sudoración & 15.0 & 55.0 & 49.6 & 0.654 & $1.244(0.479-3.233)$ \\
\hline Convulsiones & 0.8 & 100.0 & 50.0 & 1.0009 & - \\
\hline Sentirse estimulado & 16.5 & 50.0 & 50.5 & 0.969 & $0.982(0.393-2.452)$ \\
\hline Disminución del deseo sexual & 25.6 & 44.1 & 52.5 & 0.398 & $0.714(0.326-1.562)$ \\
\hline $\begin{array}{l}\text { Disminución o ausencia de } \\
\text { orgasmo }\end{array}$ & 10.5 & 35.7 & 52.1 & 0.246 & $0.511(0.162-1.614)$ \\
\hline Retardo en la eyaculación $\dagger$ & 23.3 & 57.1 & 43.5 & 0.6759 & $1.733(0.314-9.573)$ \\
\hline $\begin{array}{l}\text { Disfunción eréctil (impotencia } \\
\text { sexual) } \dagger\end{array}$ & 6.7 & 50.0 & 46.4 & 1.0009 & $1.154(0.065-20.342)$ \\
\hline $\begin{array}{l}\text { Retraso o suspensión de la } \\
\text { menstruación } \ddagger\end{array}$ & 6.8 & 71.4 & 50.0 & 0.438 & $2.500(0.462-13.521)$ \\
\hline $\begin{array}{l}\text { Contractura de alguno de sus } \\
\text { miembros }\end{array}$ & 21.1 & 39.3 & 53.3 & 0.187 & $0.566(0.242-1.324)$ \\
\hline Otros & 6.8 & 77.8 & 48.4 & 0.165 & $3.733(0.746-18.686)$ \\
\hline
\end{tabular}

* Análisis realizados sobre el total de pacientes $(\mathrm{N}=133)$ a menos que se indique lo contrario con los símbolos $\dagger$ o $\ddagger$ $\dagger$ Análisis realizado sobre el total de varones $(\mathrm{N}=133)$.

† Análisis realizado sobre el total de mujeres $(\mathrm{N}=133)$.

$\S$ Chi cuadrado excepto los valores marcados con $\mathbb{1}$

II Prueba exacta de Fisher de 2 colas. 
de la medicación antidepresiva y en el pronóstico de la depresión ha sido encontrada también en otros estudios. ${ }^{13,14,15}$ Igualmente hay estudios que han encontrado asociación entre incumplimiento e inefectividad del tratamiento. ${ }^{6,78}$ Lo mismo se puede decir sobre las consultas frecuentes con los pacientes. ${ }^{48}$

Hay otras variables como: frecuencia de la administración de la droga (una sola dosis versus viarias), la hora que la medicación es tomada y la polifarmacia que han sido asociadas a cumplimiento del tratamiento antidepresivo, ${ }^{21}$ pero nuestro estudio no ha podido confirmar estos resultados. En relación a la frecuencia de administración de la droga, nuestros resultados son comparables al estudio de Myers, ${ }^{49}$ a diferencia de que en nuestro estudio no hubo posibilidad de que los pacientes pudieran escoger la frecuencia de administración que querían. Igualmente otras variables como: comorbilidad asociada a la depresión (distimia, desorden de ansiedad y abuso de sustancias) y severidad de los síntomas depresivos que también han sido asociadas a incumplimiento del tratamiento antidepresivo, ${ }^{39,41,41,42}$ no han sido confirmadas en nuestro estudio.

\section{Factores psicosociales, efectos colaterales y cumplimiento de la medicación}

Este rubro se evaluó a través de dos cuestionarios: uno referido a aspectos psicosociales (entre ellos educaciones, tabla 7) y el otro relacionado directamente con efectos colaterales (tabla 8). Con respecto a aspectos psicosociales se encontró asociación con: falta de una explicación clara por parte del médico de cómo debía tomar la medicación, maltrato del médico, creencia de que el medicamento no ayuda o no es beneficioso, temor a hacerse dependiente de la medicación, considerar que la medicación no es necesaria para su tratamiento, considerar que es suficiente tomar la medicación hasta que uno se sienta bien y el costo de la medicación. La gran parte de estos hallazgos, que tienen que ver con aspectos educacionales, culturales corroboran lo encontrado en la literatura, lo cual ha sido expuesto en la introducción de este artículo. $6,7,8,9,10,11,12,13,14,15,16,17,18,19,48$

Con respecto a efectos colaterales, solamente se asociaron a incumplimiento: cefalea, insomnio y dolor abdominal. Esto dista de lo encontrado en la literatura, donde los efectos colaterales que se asocian más a incumplimiento son: sedación y sequedad de boca ${ }^{29}$ aumento de peso $^{32}$ y disfunciones sexuales. $^{33,34,35,36,37,38}$ Curiosamente cefalea, insomnio y dolor abdominal, son efectos colaterales que se presentan mayormente con ISRS, donde la literatura reporta mejor cumplimiento en comparación con antidepresivos tricíclicos. ${ }^{23,24,25,26,27,28,50}$ No ha sido posible realizar comparaciones entre los diferentes antidepresivos porque la cantidad de pacientes por antidepresivo era muy pequeña.

\section{Otros resultados}

Frente a la pregunta: ¿cuál fue la causa principal por la que dejó la medicación?, la gran mayoría respondió: el costo de la medicación, lo que está en relación con los bajos ingresos económicos que tiene nuestra población. Se trató en este estudio que la población escogida fuera lo más homogénea posible, por ello se tomó el INSM "HDHN" que atienda la demanda del cono norte de Lima, el HHU que atienda la demanda del cono este, el HAMA que atiende la demanda de cono sur y la CRP, que si bien es una clínica privada, el programa de despistaje gratuito está abierto a toda la población y acuden personas de diferentes estratos.

Por otro lado se pudo observar que los pacientes incumplidores presentan más pro- 
babilidad de recaídas que los cumplidores, lo que es corroborado por la literatura. ${ }^{2}$

\section{Comentarios adicionales}

Es importante comentar algunos sesgos que podrían tener el estudio. El estudio ha sido realizado en una población de consulta ambulatoria, por lo que los resultados no pueden generalizarse necesariamente a la población general. El estudio ha sido abierto, en un corto período de tiempo. Si se hubiera hecho antes, algunos incumplidores podrían haber sido cumplidores. Igualmente si se hubiera hecho después, podría esperarse de que un grupo de los cumplidores se vuelva incumplidor en el tiempo. Parte de los cumplidores con el último antidepresivo utilizado, fueron incumplidores con antidepresivos previos. Esto se obtuvo en las entrevistas, pero no fue posible introducir estos datos en el diseño del estudio. No todos los pacientes toman la medicación de marca, un grupo importante toma copias o genéricos, o combinan las diversas variedades.

\section{CONCLUSIONES}

1. En nuestro estudio, los incumplidores del tratamiento antidepresivo presentan más posibilidades de recaídas.

2. Las variables que se asociaron significativamente con cumplimiento del tratamiento fueron: psicoterapia complementaria, respuesta al tratamiento, consultas frecuentes y periódicas con su médico, falta de una explicación clara por parte del médico de cómo debía tomar la medicación, maltrato del médico, creencia de que el medicamento no ayuda o no es beneficioso, temor a hacerse dependiente de la medicación, considerar que la medicación no es necesaria para su tratamiento, considerar que es suficiente tomar la medicación hasta que uno se sienta bien, costo de la medicación y algunos efectos colaterales como cefalea, insomnio y dolor abdominal.

3. Los pacientes incumplidores consideraron que el costo de la medicación era la principal causa por la que habían dejado de tomar el tratamiento.

\footnotetext{
Agradecimientos por orden alfabético:

Dra. Elena Alza, Dr. Raúl Díaz, Dr. Luis Feijoo, Lic. Rosario Fernández Maldonado, Dr. Enrique Galli, Dr. Hever Krüger, Dr. Guido Mazzotti, Dr. Jesús Mendoza, Dr. Jorge Pizarro, Dr. Javier Saavedra, Dr. José Carlos San Martín, Dr. Noé Yactayo.
} 


\section{BIBLIOGRAFÍA}

1. Mazzotti G. Cumplimiento en el tratamiento de la depresión. Acta. Suplementos Serie Psicofarmacológica. 1998, May (1): 23-27.

2. Melfi C, Chawla A, Croghan T, Hanna M, Kennedy S, Sredl K. The effects of adherence to antidepressant Treatment Guidelines on Relapseand Recurrence of Depression. Arch Gen Psychiatry. 1998; 55: 1128-1132.

3. Fawcett J. Compliance: definitions and key issues. J. Clin Psychiatry 1995; 56 (Suppl 1): 4-8.

4. Damrosch S. Facilitating adherence to preventive and treatment regimens. En Wedding D (editors). Behavior and Medicine. St Louis, Mosby, 1995.

5. Lin EH, Von Korf M, Katon W, Bush T, Simon GE, Walker E, Robinson P. The role of the primary care physician in patients' adherence to antidepressant therapy. Med Care 1995; 33:67-74.

6. Delgado PL. Approaches to the enhancement of patient adherence to antidepressant medication treatment. J Clin Psychiatry 2000; 61 (Suppl 2): 6-9.

7. Oliver-Martin R. Psychological factors, compliance and resistance to antidepressant treatment. Encephale 1986; 12 Spec No: 197-203.

8. Johnson DA. Depression: treatment compliance in general practice. Acta Psychiatr Scand Suppl 1981; 290:447-53.

9. Seltzer A, Roncari I, Garfinkel P. Effectopatient education on medication compliance. Can J Psychiatry 1980; 25:968-45.
10. Knight JR, Campell AJ, Williams SM, Clark DW. Knowlegdgeable non-compliance with particular reference to non-steroidal antiinflamatory and antidepressant drugs. J. Clin Pharm Ther 1991; 16:131-7.

11. Altamura AC, Mauri M. Plasma concentrations, information and therapy adherence during long-term treatment with antidepressants. Br J. Clin Pharmacol 1985; 20:714-6.

12. Lin EH, Von Korff M, Katon W, Bush T, Simon GE, Walker E, Robinson P. The rol of the primary caree physician in patients' adherence to antidepressant therapy. Med Care 1995; 33:67-74.

13. Lóo H, Brochier T. Long-term treatment with antidepressive drugs. Ann Med Psychol (Paris) 1995; 153:190-6.

14. Ravndal E, Vavndal E, Vaglum P. Selfreported depression as a predictor of dropout in a hierarchical therapeutic community. J. Subst Abuse Treat, 1994 Sepoct, 11:5,471-9.

15. Paykel ES. Psychotherapy, medication combinations, and compliance. J Clin Psychiatry 1995; 56 (Suppl 1): 24-30.

16. Frank E, Kupfer DJ, Siegel LR. Alliance not compliance: a philosophy of outpatient care. J Clin Psychiatry 1995; 56 (Suppl 1), 11-6.

17. Stimmel GI. How to counsel patients about depression and its treatment. Pharmacotherapy. 1995 Nov-Dec; 15 (6 Pt 2): 100S-104S.

18. Robinson P, Katon W, Von Korff M, Bush T, Simon G, Lin E, Walker E. The edu- 
cation of depressed primary care patients: what do patients think of interactive booklets and a video? J Clin Pact 1997; 44(6): $562-571$.

19. Kinzie JD, Leung P, Boehnlein JK, Fleck J. Antidepressant blood leevels in Southeast Asians. Clinical and cultural implications. J Nerv Ment Dis 1987;175(8):480-5.

20. Hasan Mk, Mooney RP. Once-a-day drug regimen for psychiatric patients. Am Fam Psychopharmacol 1986:6(3): 144-9.

21. Cohen I, Rogers P, Burke V, Beilin LJ. Predictors of medication use, compliance and symptoms of hypotension in a community-based sample of elderly men and women. J Clin Pharm Ther 1998; 23(6): 423-32.

22. Blackwell B. Antidepressant drugs: side effects and compliance. J Clin Psychiaty. 1982; 43(11):14-21.

23. Szegedi A, Wetzel H, Angersbach D, Dunbar GC, Schwarze H, Philipp M, Benkert O. A double-blind study comparing paroxetine and maprotiline in depressed outpatients. Pharmacopsychiatry 1997 May; 30(3):97-105.

24. Mazurek J, Kiejna A. Cost analysis of treating. Comparison of tricyclic antidepressants and selective serotonine reuptake inhibitors. Psychiatr Pol. 1996 JanFeb; 30(1): 87-98.

25. Fontaine R, Ontiveros A, Elie R, KenslerTT, Roberta DL, Kaplita S, Ecker JA, Faludi G. A double-blind comparison of nefazodone, imipramine, and placebo in major depression. J Clin Psychiatry. 1994 Jun;55(6):234-41.

26. Anderson MI, Tomenson BM. Treatment discontinuation with selective serotonin reuptake inhibitors acompared with tricyclic antidepressants: a meta-analysis. BMJ. 1995 Jun 3; 310(6992):1433-8.

27. Hotopf M, Hardy R, Lewi G. Discontinuation rates of SSRIs and tricyclic antidepressants: a meta-analysis and investigation of heterogeneity. Br J Psychiatry. 1997 Feb; 170:120-7.

28. Martin RM, Hilton SR, Kerry SM, Richards NM. General practitioners' perceptions of the tolerability of antidepressant drugs: a comparison of selective serotonin reuptake inhibitors and tricyclic antidepressants. BMJ 197 Mar 1; 314 (7081):646-51.

29. Lader M. The problems of safety and compliance with conventional antidepressant drugs. Acta Psychiatr Scand. 1983, 308 (Suppl), 91-5.

30. Tollefson GD. Adverse drug reactions/ interactions in maintenance therapy. J Clin Psychiatry. 1993,54 (Suppl), 48-58.

31. Montgomery SA, Kasper S. Comparison of compliance between serotonin reuptake inhibitors and tricyclic antidepressants: a meta-analysis. Int Clin Psychopharmacol. 1995,9 (Suppl 4), 33-40.

32. Garland EJ, Remick RA, Zis AP. Weight gain with antidepressants and lithium. J Clin Psychopharmacol. 1998,8(5), 323-30.

33. Rothschild AJ. Sexual side effects of antidepressants. I Clin Psychiatry 2000; 61 Suppl 11:28-36.

34. Harrison WM, Rabkin JG, Ehrhardt AA, Steart JW, McGrath PJ, Ross D, Quitkin FM. Effects of antidepressant medication on sexual function: a controlled study. J Clin Psychopharmacol. 1986, 6(3), 144-9.

35. Balon R, Yeragani VK, Pohl R, Ramesh C. Sexual dysfunction during antidepressant 
treatment. J Clin Psychiatry. 1993, 54 (6), 209-12.

36. Baier D, Philipp M. Modificaiton of sexual functions by antidepressants. Fortschr Neurol Psychiatr. 1994,62 (1), 14-21.

37. Koutouvidis H, Pratikakis M, Fotiadou A. The use of mirtazapine in a group of 11 patients folloing poor compliance to selectiveserotonin reuptake inhibitor treatment due to sexual dysfunction. Int Clin Psychopharmacol 1999; 14(4):253-5.

38. Phillips RL, Slaughter JR. Depression and sexual desire. Am Fam Physician 2000 Aug 15;62(4):782-6.

39. Hellerstein DJ, Yanowitch P, Rosenthal J, Hemlock C, Kasch K, Samstag LW, Winston A. Long-term treatment of double depression: a preliminary study with serotonergic antidepressants. Prog Neuropsychopharmacol Biol Psychiatry. 1994 Jan; 18(1):139-147.

40. Loebel AD, Hyde TS, Dunner DL. Early placebo response in anxious and depressed patients. J Clin Psychiatry, 1996 May, 47:5, 230-3.

41. Araujo L, Goldberg P, Eyma J, Madhusoodanan S, Buff DD, Shamim K, Brenner R. The effect of anxiety and depression on completion/withdrawal status in patients admited to substance abuse detoxification program. J Subst Abuse Treat, 1996 Jan-Feb, 13:1,61-6.

42. Hooper SC, Vaughan KJ, Tennant CC, Perz JM. Major depression and refusal of life-sustaining medical treatment in the elderly. Med J Aust 1996 Oct 21; 165 (8):416-9.
43. Sherrill JT, Frank E, Geary M, Stack JA, Renolds CF. Psychoeducational workshops for elderly patients with recurrent major depression and their families. Psychiatr Serv. 1997 Jan;48(1): 76-81.

44. Natsume M, Ohta Y, Fujii H, Asao H. Studies on the occupational maladjustment syndrome. With reference ot the survery of examinations etiological factors and treatment. Sangyo Igaku, 1982 Sep,24:5, 455-64.

45. Sullivan MD, Youngner SJ. Depression, competence, and the right to refuse lifesaving treatment. Am J Psychiatry. 1994 Jul: 151(7):971-8.

46. Demyttenaere K, Van Ganse E, Gregoire J, Gaens E, Mesters P. Compliance in depressed patients treated with fluoxetine or amitriptyline. Belgian Compliance Study Group. Int Clin Psychopharmacol 1998: 13(1):11-7.

47. Cramer JA, Rosenheck R. Compliance with medication regimens for mental and physical disorders. Psychiatr Serv 1998; 49(2); 196-201.

48. Katon W, Von Korff M, Lin E, Walker E, Simon GE, Bush T, Robinson P, Russo J. Collaborative management to achieve treatment guidelines. Impact on depression in primary care. JAMA 1995;273(13): 1026-31.

49. Myers DE, Branthaite A. Out-patient compliance ith antidepressant medication. $\mathrm{Br} \mathrm{J}$ Psychiatry 1992; 160:83-6.

50. Stokes PE, Holtz A. Fluoxetine tenth anniversary update: the progress continues. Clin Ther. 1997, 19(5),1135-1249. 